\title{
Light charged Higgs boson with dominant decay to quarks and its search at the LHC and future colliders
}

\author{
A. G. Akeroyd, ${ }^{*}$ Stefano Moretti, ${ }^{\dagger}$ and Muyuan Song \\ School of Physics and Astronomy, University of Southampton, \\ Highfield, Southampton SO17 1BJ, United Kingdom
}

(Received 24 October 2018; published 18 December 2018)

\begin{abstract}
The possibility of a light charged Higgs boson $H^{ \pm}$that decays predominantly to quarks ( $c s$ and/or $c b$ ) and with a mass in the range $80 \mathrm{GeV} \leq m_{H^{ \pm}} \leq 90 \mathrm{GeV}$ is studied in the context of three-Higgs-doublet models (3HDMs). At present, the Large Hadron Collider (LHC) has little sensitivity to this scenario, and currently the best constraints are from LEP2 and Tevatron searches. The branching ratio of $H^{ \pm} \rightarrow c b$ can be dominant in two of the five types of 3HDM, and we determine the parameter space where this occurs. The decay $H^{ \pm} \rightarrow c b$ has recently been searched for at the LHC for the first time, and with increased integrated luminosity, one would expect sensitivity to the region $80 \mathrm{GeV} \leq m_{H^{ \pm}} \leq 90 \mathrm{GeV}$ due to the smaller backgrounds with respect to $H^{ \pm} \rightarrow$ cs decays.
\end{abstract}

DOI: 10.1103/PhysRevD.98.115024

\section{INTRODUCTION}

In 2012, the ATLAS and CMS Collaborations of the Large Hadron Collider (LHC) announced the discovery of a new particle with a mass of around $125 \mathrm{GeV}[1,2]$. The current measurements of its properties are in very good agreement (within experimental error) with those of the Higgs boson of the standard model (SM), and measurements suggest that it has a spin of zero. Five decay channels $(\gamma \gamma, Z Z, W W, \tau \tau$, and $b b)$ have now been observed with a statistical significance of greater than $5 \sigma$ (e.g., see [3]). The measured branching ratios (BRs) are in agreement with those predicted for the SM Higgs boson. Moreover, the main four production mechanisms (gluon-gluon fusion, vector boson $(W / Z)$ fusion, associated production with a vector boson, and associated production with top quarks) have been observed, with no significant deviation from the cross sections of the SM Higgs boson.

The simplest assumption is that the observed $125 \mathrm{GeV}$ boson is the (solitary) Higgs boson of the SM. However, it is possible that it is the first scalar to be discovered from a nonminimal Higgs sector, which contains additional scalar isospin doublets or higher representations such as scalar isospin triplets. In such a scenario, future measurements of

\footnotetext{
*a.g.akeroyd@soton.ac.uk

†.Moretti@soton.ac.uk

ms32g13@ soton.ac.uk
}

Published by the American Physical Society under the terms of the Creative Commons Attribution 4.0 International license. Further distribution of this work must maintain attribution to the author(s) and the published article's title, journal citation, and DOI. Funded by SCOAP ${ }^{3}$. the BRs of the $125 \mathrm{GeV}$ boson could show deviations from those of the SM Higgs boson. There is also the possibility of discovering additional neutral scalars, or physical charged scalars $\left(H^{ \pm}\right)$. In the context of a two-Higgs-doublet model (2HDM), the lack of observation of an $H^{ \pm}$at the LHC rules out parameter space of $\tan \beta$ (from the Yukawa coupling) and $m_{H^{ \pm}}$, where $\tan \beta=v_{2} / v_{1}$, and $v_{1}$ and $v_{2}$ are the vacuum expectation values (VEVs) of the two Higgs doublets, respectively (for reviews, see e.g., [4,5]).

In a three-Higgs-doublet model (3HDM), the Yukawa couplings of the two charged scalars depend on the four free parameters $(\tan \beta, \tan \gamma, \theta$, and $\delta)$ of the unitary matrix that rotates the charged scalar fields in the weak eigenbasis to the physical charged scalar fields. As pointed out in previous works [6-10], in a $3 \mathrm{HDM}$ there is a phenomenologically attractive possibility of an $H^{ \pm}$being light $\left(m_{H^{ \pm}}<m_{t}\right)$ and having a large BR for the decay channel $H^{ \pm} \rightarrow c b$, a scenario which would not be expected in a 2HDM with natural flavor conservation (NFC) [11] due to the stringent bounds from the decay $b \rightarrow s \gamma$. A search for $H^{ \pm} \rightarrow c b$ decays originating from $t \rightarrow H^{ \pm} b$ has recently been performed at the LHC [12]. The only study of the BRs of the two $H^{ \pm} \mathrm{S}$ in 3HDMs (with NFC) as functions of the above four parameters was in Ref. [10]. However, this work did not fully study the dependence of the BRs on the parameter space. We perform the first comprehensive study of the BRs of the lightest $H^{ \pm}$in the various 3HDMs (with NFC) as a function of the four parameters. We also study the dependence of the product $\operatorname{BR}\left(t \rightarrow H^{ \pm} b\right) \times \mathrm{BR}\left(H^{ \pm} \rightarrow c b\right)$, which gives the number of events in the search in [12]. We give emphasis to the scenario of $80 \mathrm{GeV} \leq m_{H^{ \pm}} \leq 90 \mathrm{GeV}$ and a large $\operatorname{BR}\left(H^{ \pm} \rightarrow c s / c b\right)$ for which detection is 
currently challenging at the LHC, but prospects with the anticipated integrated luminosities are more promising.

This work is organized as follows. In Sec. II, we give an introduction to the phenomenology of the lightest $H^{ \pm}$in 3HDMs with NFC. In Sec. III, the searches for $H^{ \pm}$at past and present colliders that provide sensitivity to the region $80 \mathrm{GeV}<m_{H^{ \pm}}<90 \mathrm{GeV}$ are summarized. In Sec. IV, our results are presented, and conclusions are contained in Sec. V.

\section{THE THREE-HIGGS-DOUBLET MODEL (3HDM) WITH NFC}

In this section, the fermionic couplings of the lightest $H^{ \pm}$ in the $3 \mathrm{HDM}$ as a function of the parameters of the scalar potential are presented. The constraints on the fermionic couplings are summarized, and explicit formulae for the BRs of the decay of $H^{ \pm}$to fermions are given.

\section{A. Fermionic couplings of $\boldsymbol{H}^{ \pm}$in the $3 \mathrm{HDM}$}

In a 2HDM, the Lagrangian that corresponds to the interactions of $H^{ \pm}$with the fermions (the Yukawa couplings) can be written as follows:

$$
\begin{aligned}
\mathcal{L}_{H^{ \pm}}= & -\left\{\frac{\sqrt{2} V_{u d}}{v} \bar{u}\left(m_{d} X P_{R}+m_{u} Y P_{L}\right) d H^{+}\right. \\
& \left.+\frac{\sqrt{2} m_{\ell}}{v} Z_{\nu_{L}} \ell_{R} H^{+}+\text {H.c. }\right\} .
\end{aligned}
$$

Here $u(d)$ refers to the up(down)-type quarks, and $\ell$ refers to the electron, muon and tau. The imposition of NFC, which eliminates tree-level flavor changing neutral currents (FCNCs) that are mediated by scalars, is achieved by requiring that the Yukawa couplings are invariant under certain discrete symmetries (e.g., see [10] for the charge assignments of the scalar and fermion fields under the discrete symmetries). This leads to four distinct 2HDMs [13]: Type I, Type II, lepton-specific, and flipped. In Table I, the couplings $X, Y$, and $Z$ in the four distinct 2HDMs are given. The Lagrangian in Eq. (1) also applies to the lightest $H^{ \pm}$of a $3 \mathrm{HDM}$, with the $X, Y$, and $Z$ couplings being functions of four parameters of a unitary matrix $U$. This matrix $U$ connects the charged scalar fields in the

TABLE I. The couplings $X, Y$, and $Z$ in the Yukawa interactions of $H^{ \pm}$in the four versions of the $2 \mathrm{HDM}$ with NFC.

\begin{tabular}{lrrr}
\hline \hline & $X$ & $Y$ & \multicolumn{1}{c}{$Z$} \\
\hline Type I & $-\cot \beta$ & $\cot \beta$ & $-\cot \beta$ \\
Type II & $\tan \beta$ & $\cot \beta$ & $\tan \beta$ \\
Lepton-specific & $-\cot \beta$ & $\cot \beta$ & $\tan \beta$ \\
Flipped & $\tan \beta$ & $\cot \beta$ & $-\cot \beta$ \\
\hline \hline
\end{tabular}

weak eigenbasis $\left(\phi_{1}^{ \pm}, \phi_{2}^{ \pm}, \phi_{3}^{ \pm}\right)$with the physical scalar fields $\left(H_{1}^{ \pm}, H_{2}^{ \pm}\right)$and the charged Goldstone boson $G^{ \pm}$as follows:

$$
\left(\begin{array}{c}
G^{+} \\
H_{1}^{+} \\
H_{2}^{+}
\end{array}\right)=U\left(\begin{array}{c}
\phi_{1}^{+} \\
\phi_{2}^{+} \\
\phi_{3}^{+}
\end{array}\right) .
$$

We take $H_{1}^{ \pm}$as the lighter of the two charged Higgs bosons, and from now on it is referred to as $H^{ \pm}$with the following couplings [14]:

$$
X=\frac{U_{d 2}^{\dagger}}{U_{d 1}^{\dagger}}, \quad Y=-\frac{U_{u 2}^{\dagger}}{U_{u 1}^{\dagger}}, \quad Z=\frac{U_{\ell 2}^{\dagger}}{U_{\ell 1}^{\dagger}} .
$$

The values of $d, u$, and $\ell$ in these matrix elements are given in Table II and depend on which of the five distinct 3HDMs is under consideration. Taking $d=1, u=2$, and $\ell=3$ means that the down-type quarks receive their mass from the vacuum expectation value $v_{1}$, the up-type quarks from $v_{2}$, and the charged leptons from $v_{3}$ (this choice is called the "democratic 3HDM"). The other possible choices of $d$, $u$, and $\ell$ in a $3 \mathrm{HDM}$ are given the same names as the four types of 2HDM. The couplings of the $H_{2}^{ \pm}$(i.e., the heavier charged scalar) are obtained from Eq. (3) by making the replacement $2 \rightarrow 3$ in the numerators of $X, Y$, and $Z$. We will not study these couplings for $H_{2}^{ \pm}$because our focus will be on $H^{ \pm}$in the range $80 \mathrm{GeV}<m_{H^{ \pm}}<90 \mathrm{GeV}$.

The matrix $U$ can be written explicitly as a function of four parameters $\tan \beta, \tan \gamma, \theta$, and $\delta$, where

$$
\tan \beta=v_{2} / v_{1}, \quad \tan \gamma=\sqrt{v_{1}^{2}+v_{2}^{2}} / v_{3}
$$

and $v_{1}, v_{2}$, and $v_{3}$ are the VEVs. The angle $\theta$ and phase $\delta$ can be written explicitly as functions of several parameters in the scalar potential [14]. The explicit form of $U$ is:

TABLE II. The five versions of the $3 \mathrm{HDM}$ with NFC, and the corresponding values of $u, d$, and $\ell$. Taking $u=2$ means that the up-type quarks receive their mass from the vacuum expectation value $v_{2}$, and likewise for $d$ (down-type quarks) and $\ell$ (charged leptons).

\begin{tabular}{llll}
\hline \hline & $u$ & $d$ & $\ell$ \\
\hline 3HDM (Type I) & 2 & 2 & 2 \\
3HDM (Type II) & 2 & 1 & 1 \\
3HDM (Lepton-specific) & 2 & 2 & 1 \\
3HDM (Flipped) & 2 & 1 & 2 \\
3HDM (Democratic) & 2 & 1 & 3 \\
\hline \hline
\end{tabular}




$$
\begin{aligned}
U & =\left(\begin{array}{ccc}
1 & 0 & 0 \\
0 & e^{-i \delta} & 0 \\
0 & 0 & 1
\end{array}\right)\left(\begin{array}{ccc}
1 & 0 & 0 \\
0 & c_{\theta} & s_{\theta} e^{i \delta} \\
0 & -s_{\theta} e^{-i \delta} & c_{\theta}
\end{array}\right)\left(\begin{array}{ccc}
s_{\gamma} & 0 & c_{\gamma} \\
0 & 1 & 0 \\
-c_{\gamma} & 0 & s_{\gamma}
\end{array}\right)\left(\begin{array}{ccc}
c_{\beta} & s_{\beta} & 0 \\
-s_{\beta} & c_{\beta} & 0 \\
0 & 0 & 1
\end{array}\right) \\
& =\left(\begin{array}{ccc}
s_{\gamma} c_{\beta} & s_{\gamma} s_{\beta} & c_{\theta} \\
-c_{\theta} s_{\beta} e^{-i \delta}-s_{\theta} c_{\gamma} c_{\beta} & c_{\theta} c_{\beta} e^{-i \delta}-s_{\theta} c_{\gamma} s_{\beta} & s_{\theta} s_{\gamma} \\
s_{\theta} s_{\beta} e^{-i \delta}-c_{\theta} c_{\gamma} c_{\beta} & -s_{\theta} c_{\beta} e^{-i \delta}-c_{\theta} c_{\gamma} s_{\beta} & c_{\theta} s_{\gamma}
\end{array}\right) .
\end{aligned}
$$

Here $s$ and $c$ denote the sine or cosine of the respective angle. Hence, the functional forms of $X, Y$, and $Z$ in a $3 \mathrm{HDM}$ depend on four parameters. This is in contrast to the analogous couplings in the $2 \mathrm{HDM}$ for which $\tan \beta$ is the only free coupling parameter.

\section{B. Constraints on the couplings $X, Y$, and $Z$}

The couplings $X, Y$, and $Z$ (and their combinations) are constrained from various low-energy processes. Detailed studies in the context of the Aligned 2HDM (for which the couplings of $H^{ \pm}$are also given by $X, Y$, and $Z$ ) can be found in Refs. $[15,16]$. These constraints can be applied to the lightest $H^{ \pm}$of a $3 \mathrm{HDM}$ provided that the contribution to a given process from the $H_{2}^{ \pm}$is considerably smaller (e.g., if $m_{H_{2}^{ \pm}} \gg m_{H^{ \pm}}$). In this work, we assume that any contribution from $H_{2}^{ \pm}$is sub-dominant and can be neglected to a good approximation. We summarize here the bounds (which are also summarized in [14]) that we will use in our numerical analysis.

The coupling $Y$ is constrained from the process $Z \rightarrow b \bar{b}$ from LEP data. For $m_{H^{ \pm}}$around $100 \mathrm{GeV}$ (on which we focus) the constraint is roughly $|Y|<1$ (assuming $|X| \leq 50$, so that the dominant contribution is from the $Y$ coupling). The coupling $X$ is also constrained from $Z \rightarrow b \bar{b}$, but the constraints from this process are weaker than those from $t \rightarrow H^{ \pm} b$ (which will be studied later in this work).

From the rare decay $b \rightarrow s \gamma$ a constraint on the combination $\operatorname{Re}\left(X Y^{*}\right)$ is given by

$$
-1.1 \leq \operatorname{Re}\left(X Y^{*}\right) \leq 0.7 .
$$

This constraint was derived in [16] for $m_{H^{ \pm}}=100 \mathrm{GeV}$, and is an approximation for the case when i) the contribution from $|Y|^{2}$ can be neglected (which is a fairly good approximation because $|Y|<1)$ and ii) $\operatorname{Im}\left(X Y^{*}\right)$ is small (which is a good approximation, as shown shortly below). Detailed constraints on the $H^{ \pm}$contribution to $b \rightarrow s \gamma$ in the Aligned 2HDM without this approximation can be found in [15]. Other works are usually in the context of the 2HDM with NFC [17-22].

In a 3HDM, one would have contributions to $b \rightarrow s \gamma$ from both $H^{ \pm}$and $H_{2}^{ \pm}$. The only study of the prediction for
$\mathrm{BR}(b \rightarrow s \gamma)$ in $3 \mathrm{HDM}$ s to next-to-leading order accuracy is in [10]. It was shown there that there exists parameter space for which $H^{ \pm}$can be of the order of $80 \mathrm{GeV}$ even for Type II and flipped structures (which would not be possible in the 2HDM with these structures). This is due to the additional presence of $H_{2}^{ \pm}$and the larger number of parameters in the couplings $X$ and $Y$ with respect to the $2 \mathrm{HDM}$ with NFC. In our numerical analysis for the BRs of $H^{ \pm}$, we will use the allowed range given in Eq. (6) in order to find the regions of $\tan \beta, \tan \gamma, \theta$, and $\delta$ that satisfy the $b \rightarrow s \gamma$ constraint. Although Eq. (6) neglects the contribution from $H_{2}^{ \pm}$we will take Eq. (6) as being representative of the $b \rightarrow s \gamma$ constraint in 3HDMs. The true region allowed by $b \rightarrow s \gamma$ (to next-to leading order accuracy, as done in [10]) would presumably be shifted somewhat from the regions allowed by Eq. (6). We argue later that we would not expect this to significantly alter our qualitative results.

The electric dipole moment of the neutron gives the following constraint on $\operatorname{Im}\left(X Y^{*}\right)$ [16]:

$$
\left|\operatorname{Im}\left(X Y^{*}\right)\right| \leq 0.1 \text {. }
$$

This bound is for $m_{H^{ \pm}}=100 \mathrm{GeV}$ and is an order-ofmagnitude estimate. There are also the constraints $|Z| \leq 40$ and $|X Z| \leq 1080$, both for $m_{H^{ \pm}}=100 \mathrm{GeV}$. In our numerical analysis, we will respect all these constraints.

\section{The Branching Ratios of $\boldsymbol{H}^{ \pm}$}

We will only consider the decays of $H^{ \pm}$to fermions. If there exists a neutral scalar (e.g., a $C P$-even $h^{0}$ or a $C P$-odd $A^{0}$ ) that is lighter than $H^{ \pm}$then the decay channel $H^{ \pm} \rightarrow$ $h^{0} W^{*}$ and/or $H^{ \pm} \rightarrow A^{0} W^{*}$ would be open and can be sizeable (or even dominant) [9,23-29]. We assume that these decays are negligible, and this is most easily achieved by taking $m_{A^{0}}, m_{h^{0}}>m_{H^{ \pm}}$. In a $3 \mathrm{HDM}$, the expressions for the partial widths of the decay modes to fermions of $H^{ \pm}$ are:

$$
\begin{gathered}
\Gamma\left(H^{ \pm} \rightarrow \ell^{ \pm} \nu\right)=\frac{G_{F} m_{H^{ \pm}} m_{\ell}^{2}|Z|^{2}}{4 \pi \sqrt{2}}, \\
\Gamma\left(H^{ \pm} \rightarrow u d\right)=\frac{3 G_{F} V_{u d} m_{H^{ \pm}}\left(m_{d}^{2}|X|^{2}+m_{u}^{2}|Y|^{2}\right)}{4 \pi \sqrt{2}} .
\end{gathered}
$$


In the expression for $\Gamma\left(H^{ \pm} \rightarrow u d\right)$, the running quark masses should be evaluated at the scale of $m_{H^{ \pm}}$, and there are QCD vertex corrections which multiply the partial widths by $\left(1+17 \alpha_{s} /(3 \pi)\right)$. A study of the BRs as a function of $|X|,|Y|$, and $|Z|$ was first given in [7] and more recently in [9]. For $|X| \gg|Y|,|Z|$ the decay channel $\operatorname{BR}\left(H^{ \pm} \rightarrow c b\right)$ can dominate (which was first mentioned in [6]), reaching a maximum of $\sim 80 \%$. In contrast, in a 2HDM with NFC the only model which contains a parameter space for a large $\operatorname{BR}\left(H^{ \pm} \rightarrow c b\right)$ with $m_{H^{ \pm}}<$ $m_{t}$ is the flipped model (a possibility mentioned in $[6,7]$ and studied in more detail in [30,31]), However, in this case the $b \rightarrow s \gamma$ constraint would require $m_{H^{ \pm}}>500 \mathrm{GeV}$ [22] for which $H^{ \pm} \rightarrow t b$ would dominate.

The first study of the dependence of the BRs of $H^{ \pm}$in 3HDMs in terms of the parameters $\tan \beta, \tan \gamma, \theta$, and $\delta$ was given in [10]. However, this work did not fully study the dependence of the BRs on the parameter space (i.e., $\delta=0$, $\theta=-\pi / 4$, and $\tan \beta=2(5)$ was taken as a representative choice), and showed the BRs as a function of $\tan \gamma$ only. Moreover, in [10] the dependence of the BRs on the model parameters was carried out in the Higgs basis, and so the parameters $\tan \beta, \tan \gamma, \theta$, and $\delta$ used in that work are not equivalent to the corresponding parameters in this work.

We now briefly mention other models in which a large $\operatorname{BR}\left(H^{ \pm} \rightarrow c b\right)$ is possible, although in this work we will just study the 3HDMs with NFC. The $X, Y$, and $Z$ couplings of $H^{ \pm}$in the Aligned 2HDM [32] (which does not have NFC, but instead eliminates scalar FCNCs at tree level by taking certain Yukawa matrices to be proportional to each other) are functions of five parameters. Consequently, $|X| \gg|Y|,|Z|$ can be realized and a large $\mathrm{BR}\left(H^{ \pm} \rightarrow c b\right)$ is possible [9]. In the 2HDM (Type III), in which fermions receive their masses from both VEVs (and scalar FCNCs are present at tree level), the Yukawa couplings of $H^{ \pm}$depend on more parameters than in the Aligned 2HDM and thus a large $\operatorname{BR}\left(H^{ \pm} \rightarrow c b\right)$ can be obtained [33]. Similar comments apply to a four-Higgsdoublet model [14]. In models for which $X, Y$, and $Z$ depend on several parameters, one expects some parameter space for a large $\operatorname{BR}\left(H^{ \pm} \rightarrow c b\right)$ for $m_{H^{ \pm}}<m_{t}$, while satisfying the $b \rightarrow s \gamma$ constraint.

\section{SEARCHES FOR $\boldsymbol{H}^{ \pm}$IN THE REGION $80 \mathrm{GeV} \leq \boldsymbol{m}_{\boldsymbol{H}^{ \pm}} \leq 90 \mathrm{GeV}$}

We focus on the scenario of $H^{ \pm}$being lighter than the top quark. There have been searches for $H^{ \pm}$in the region $80 \mathrm{GeV} \leq m_{H^{ \pm}} \leq 90 \mathrm{GeV}$ at LEP2, Tevatron and the LHC. However, the sensitivity to this mass region is often inferior to that for $90 \mathrm{GeV} \leq m_{H^{ \pm}} \leq 160 \mathrm{GeV}$ because of the large backgrounds from $W$ decays. We pay particular attention to the region of $80 \mathrm{GeV} \leq m_{H^{ \pm}} \leq 90 \mathrm{GeV}$, and in the following we discuss the searches for $m_{H^{ \pm}}<m_{t}$ at each of these colliders.

\section{A. Tevatron searches}

At the Fermilab Tevatron the production mechanism is $p \bar{p} \rightarrow t \bar{t}$, where one top quark decays conventionally via $t \rightarrow W b$ and the other top quark decays via $t \rightarrow H^{ \pm} b$. Taking $\left|V_{t b}\right|=1$ one has the following expressions for the decays of a top quark to a $W$ boson or an $H^{ \pm}$:

$$
\begin{aligned}
\Gamma\left(t \rightarrow W^{ \pm} b\right) & =\frac{G_{F} m_{t}}{8 \sqrt{2} \pi}\left[m_{t}^{2}+2 M_{W}^{2}\right]\left[1-M_{W}^{2} / m_{t}^{2}\right]^{2}, \\
\Gamma\left(t \rightarrow H^{ \pm} b\right) & =\frac{G_{F} m_{t}}{8 \sqrt{2} \pi}\left[m_{t}^{2}|Y|^{2}+m_{b}^{2}|X|^{2}\right]\left[1-m_{H^{ \pm}}^{2} / m_{t}^{2}\right]^{2} .
\end{aligned}
$$

As can be seen from the above equations the $\operatorname{BR}\left(t \rightarrow H^{ \pm} b\right)$ depends on the magnitude of $|X|$ and $|Y|$. As discussed earlier, the BRs of $H^{ \pm}$depend on the relative values of $|X|,|Y|$ and $|Z|$. The search by the D0 Collaboration in [34] with $1 \mathrm{fb}^{-1}$ of data obtained the following limit in the region $80 \mathrm{GeV} \leq m_{H^{ \pm}} \leq 90 \mathrm{GeV}$ :

$\mathrm{BR}\left(t \rightarrow H^{ \pm} b\right)<0.21$ for $50 \% \leq \mathrm{BR}\left(H^{ \pm} \rightarrow c s\right) \leq 100 \%$.

In the search strategy in [34], the presence of a large $\operatorname{BR}\left(H^{ \pm} \rightarrow c s / c b\right)$ in the decay $t \rightarrow H^{ \pm} b$ would lead to a depletion in the expected number of events in the $\ell+$ jets, $\ell \ell$ and $\ell \tau$ channels $(\ell=e$ or $\mu$ ) compared to that expected from $t \bar{t} \rightarrow W^{+} W^{-} b \bar{b}$. Importantly, this "disappearance" search has sensitivity to the region $80 \mathrm{GeV} \leq m_{H^{ \pm}} \leq$ $90 \mathrm{GeV}$ and is thus an effective strategy when $\operatorname{BR}\left(H^{ \pm} \rightarrow\right.$ $c s / c b)$ is large and $m_{H^{ \pm}}$lies in the above region.

The CDF Collaboration (with $2.2 \mathrm{fb}^{-1}$ ) used a different search strategy [35] in which the signature of $H^{ \pm} \rightarrow c s$ was searched for as a peak at $m_{H^{ \pm}}$in the invariant mass distribution of the quarks that it decays to (i.e., an "appearance" search for $\left.H^{ \pm} \rightarrow c s\right)$. This technique provides limits on $\operatorname{BR}\left(t \rightarrow H^{ \pm} b\right)$ that are competitive with those in [34] for values of $m_{H^{ \pm}}$that are not in the region $80 \mathrm{GeV} \leq m_{H^{ \pm}} \leq 90 \mathrm{GeV}$. However the search provides no constraints for $80 \mathrm{GeV} \leq m_{H^{ \pm}} \leq 90 \mathrm{GeV}$ because the background from $W \rightarrow q \bar{q}$ is too large. Up to now the LHC has only carried out appearance searches for $H^{ \pm} \rightarrow c s / c b$ (see below).

\section{B. LHC searches}

The production mechanism at the LHC is $p p \rightarrow t \bar{t}$, where one top quark decays via $t \rightarrow H^{ \pm} b$ (i.e., the same mechanism at the parton level as at the Tevatron). The LHC is expected to have accumulated around $150 \mathrm{fb}^{-1}$ of integrated luminosity at $\sqrt{s}=13 \mathrm{TeV}$ by the end of the year 2018, at which point long shut down 2 will commence. 
TABLE III. Searches for $H^{ \pm}$at the LHC, using $p p \rightarrow t \bar{t}$ and $t \rightarrow H^{ \pm} b$. The given integrated luminosities are approximate. The search in [36] used $2 \mathrm{fb}^{-1}$, and the search in [37] used $13 \mathrm{fb}^{-1}$.

\begin{tabular}{lcc}
\hline \hline & ATLAS & CMS \\
\hline $7 \mathrm{TeV}\left(5 \mathrm{fb}^{-1}\right)$ & $c s[38], \tau \nu[39,40]$ & $\tau \nu[36]$ \\
$8 \mathrm{TeV}\left(20 \mathrm{fb}^{-1}\right)$ & $\tau \nu[41]$ & $c s[42], c b[12], \tau \nu[43]$ \\
$13 \mathrm{TeV}\left(36 \mathrm{fb}^{-1}\right)$ & $\tau \nu[44]$ & $\tau \nu[37,45]$ \\
\hline \hline
\end{tabular}

Various searches for the decay $t \rightarrow H^{ \pm} b$ have been carried out at the LHC, and are summarized in Table III.

\section{Decay $\mathrm{H}^{ \pm} \rightarrow \tau \nu$}

For the decay $H^{ \pm} \rightarrow \tau \nu$ there are four basic signatures, which arise from the leptonic and hadronic decays of $H^{ \pm}$ and $W^{ \pm}$. Searches for three of these signatures have been carried out with the $7 \mathrm{TeV}$ data $[36,39,40]$, which were then combined to give a limit on the product $\operatorname{BR}\left(t \rightarrow H^{ \pm} b\right) \times$ $\operatorname{BR}\left(H^{ \pm} \rightarrow \tau \nu\right)$ for a given $m_{H^{ \pm}}$. Note that ATLAS used two different search strategies $[39,40]$ that give comparable sensitivity. In $[36,39,40]$, the limit is roughly $\geq 4 \%$ for $m_{H^{ \pm}}=90 \mathrm{GeV}$, which strengthens with increasing $m_{H^{ \pm}}$ to $\geq 1 \%$ for $m_{H^{ \pm}}=160 \mathrm{GeV}$. Only the CMS search [36] presented limits $(\geq 4 \%)$ for the mass range $80 \mathrm{GeV} \leq$ $m_{H^{ \pm}} \leq 90 \mathrm{GeV}$.

In the searches for $H^{ \pm} \rightarrow \tau \nu$ with the $8 \mathrm{TeV}$ data [41,43], both the $\tau$ and the $W$ boson from $t \rightarrow W^{ \pm} b$ decay were taken to decay hadronically. This signature (of the four) offers the greatest sensitivity at present. The transverse mass of $H^{ \pm}$is calculated from its decay products of hadrons and missing energy. Both the ATLAS and CMS searches presented limits for the mass range $80 \mathrm{GeV} \leq$ $m_{H^{ \pm}} \leq 90 \mathrm{GeV}$. Limits on the product $\operatorname{BR}\left(t \rightarrow H^{ \pm} b\right) \times$ $\operatorname{BR}\left(H^{ \pm} \rightarrow \tau \nu\right)$ were obtained, being around $\geq 1 \%$ for $m_{H^{ \pm}}=80 \mathrm{GeV}$ and strengthening with increasing $m_{H^{ \pm}}$ to $\geq 0.2 \%$ for $m_{H^{ \pm}}=160 \mathrm{GeV}$.

The CMS search [37] with $13 \mathrm{TeV}$ data and $13 \mathrm{fb}^{-1}$ also used the hadronic decay of the $\tau$ from $H^{ \pm} \rightarrow \tau \nu$, and selected the hadronic decay of the $W^{ \pm}$. Similar limits to those in $[41,43]$ were obtained, but are slightly weaker for the region $80 \mathrm{GeV} \leq m_{H^{ \pm}} \leq 90 \mathrm{GeV}$. Recently a CMS search was carried out with $13 \mathrm{TeV}$ data and $36 \mathrm{fb}^{-1}$ [45], which combined separate searches for three of the four basic signatures (the case where both the $W$ and $\tau$ decay leptonically was not searched for). Significantly improved limits on $\operatorname{BR}\left(t \rightarrow H^{ \pm} b\right) \times \mathrm{BR}\left(H^{ \pm} \rightarrow \tau \nu\right)$ were obtained, ranging from $\geq 0.36 \%$ for $m_{H^{ \pm}}=80 \mathrm{GeV}$ to $\geq 0.08 \%$ for $m_{H^{ \pm}}=160 \mathrm{GeV}$.

There has been a search with the $13 \mathrm{TeV}$ data [44] from the ATLAS Collaboration using $36 \mathrm{fb}^{-1}$, with limits similar to those in [45]. In contrast to the ATLAS search with $8 \mathrm{TeV}$ data [41], both the leptonic and hadronic decays of the $W^{ \pm}$boson were considered (the $\tau$ is still taken to decay hadronically). No limits are presented for the region $80 \mathrm{GeV} \leq m_{H^{ \pm}} \leq 90 \mathrm{GeV}$, but the sensitivity to $m_{H^{ \pm}}>$ $90 \mathrm{GeV}$ has improved by a factor of approximately 5 to 10 e.g., for $m_{H^{ \pm}}=90 \mathrm{GeV}$ the limit on $\mathrm{BR}\left(t \rightarrow H^{ \pm} b\right) \times$ $\operatorname{BR}\left(H^{ \pm} \rightarrow \tau \nu\right)$ is $\geq 0.3 \%$, and with the $8 \mathrm{TeV}$ data it is $\geq 1.2 \%$.

\section{Decay $\mathrm{H}^{ \pm} \rightarrow c s / c b$}

ATLAS carried out a search for $H^{ \pm} \rightarrow c s$ [38] with $5 \mathrm{fb}^{-1}$ of data at $7 \mathrm{TeV}$, while CMS [42] carried out a search for $H^{ \pm} \rightarrow$ cs using $20 \mathrm{fb}^{-1}$ of data at $8 \mathrm{TeV}$. The $W$ boson is taken to decay leptonically. Two tagged $b$-quarks are required (which arise from the decay of the $t$-quarks), and the invariant mass distribution of the two quarks that are not $b$-tagged (i.e., the $c$ and $s$ quarks that originate from $H^{ \pm}$) is plotted. The signature of $H^{ \pm}$would be a peak at $m_{H^{ \pm}}$in this invariant mass distribution. Limits on the product $\left.\mathrm{BR}\left(t \rightarrow H^{ \pm} b\right) \times \mathrm{BR}\left(H^{ \pm} \rightarrow c s\right)\right)$ are obtained, which range from around $\geq 5 \%$ for $m_{H^{ \pm}}=90 \mathrm{GeV}$ to $2 \%$ for $m_{H^{ \pm}}=160 \mathrm{GeV}$. Note that these limits are weaker than those for $H^{ \pm} \rightarrow \tau \nu$ decay for a given $m_{H^{ \pm}}$. In the invariant mass distribution, the dominant background from $W \rightarrow q q$ decays gives rise to a peak around $80 \mathrm{GeV}$. Hence, the expected sensitivity starts to weaken significantly with decreasing $m_{H^{ \pm}}$in the region $90 \mathrm{GeV} \leq m_{H^{ \pm}} \leq 100 \mathrm{GeV}$, and there are no limits for the region $80 \mathrm{GeV} \leq m_{H^{ \pm}} \leq$ $90 \mathrm{GeV}$.

CMS carried out a search [12] for $H^{ \pm} \rightarrow c b$ decays (assuming a branching ratio of $100 \%$ ) with the leptonic decay of $W$. Signal events will have three $b$-quarks, although one (or more) might not be tagged as a $b$-quark. Two event categories were defined: i) $3 b+e^{ \pm}$, and ii) $3 b+\mu^{ \pm}$. A fitting procedure was carried out in order to correctly identify the tagged $b$-quark that arises from $H^{ \pm} \rightarrow c b$, which is then used (together with the non- $b$ tagged $c$ quark) in the invariant mass distribution of $H^{ \pm}$. Due to $\operatorname{BR}(W \rightarrow c b)$ being very small, the background to $H^{ \pm} \rightarrow c b$ decays is much smaller than that for $H^{ \pm} \rightarrow c s$. Combining both event categories results in limits on $\mathrm{BR}\left(t \rightarrow H^{ \pm} b\right) \times \mathrm{BR}\left(H^{ \pm} \rightarrow c b\right)$ of around $\geq 1.4 \%$ for $m_{H^{ \pm}}=90 \mathrm{GeV}$, which strengthens with increasing $m_{H^{ \pm}}$ to $\geq 0.5 \%$ for $m_{H^{ \pm}}=150 \mathrm{GeV}$. These limits are stronger than those for $H^{ \pm} \rightarrow c s$ decays for a given $m_{H^{ \pm}}$. Again, no limits are given in the mass range $80 \mathrm{GeV} \leq m_{H^{ \pm}} \leq$ $90 \mathrm{GeV}$, although (unlike the case for $H^{ \pm} \rightarrow c s$ ) the sensitivity does not diminish considerably in the range $90 \mathrm{GeV} \leq m_{H^{ \pm}} \leq 100 \mathrm{GeV}$.

\section{Sensitivity to $80 \mathrm{GeV} \leq \boldsymbol{m}_{H^{ \pm}} \leq 90 \mathrm{GeV}$ for future $\mathrm{LHC}$ searches for $\mathrm{H}^{ \pm} \rightarrow c s / c b$}

Given the significantly lower backgrounds for the $3 b$ signature arising from $H^{ \pm} \rightarrow c b$ decays it is hoped that 
future searches (e.g., with $150^{-1} \mathrm{fb}$ and $\sqrt{s}=13 \mathrm{TeV}$ ) will be able to set limits on $\mathrm{BR}\left(t \rightarrow H^{ \pm} b\right) \times \mathrm{BR}\left(H^{ \pm} \rightarrow c b\right)$ in the region $80 \mathrm{GeV} \leq m_{H^{ \pm}} \leq 90 \mathrm{GeV}$. Eventually, one would also expect some sensitivity in this region for the search with the $2 b$ signature (which is sensitive to $H^{ \pm} \rightarrow$ $c s / c b$ decays) with $150^{-1} \mathrm{fb}$ and above. However, the limits would (most likely) be inferior to those in the $3 b$ channel for a given luminosity.

As mentioned earlier, the Tevatron strategy of a disappearance search for $H^{ \pm} \rightarrow c s / c b$ has not yet been attempted at the LHC. A dedicated disappearance search at the LHC would be likely to improve on the Tevatron limit on $\operatorname{BR}\left(t \rightarrow H^{ \pm} b\right) \times \mathrm{BR}\left(H^{ \pm} \rightarrow c s / c b\right)$ of $20 \%$ [34] for $80 \mathrm{GeV} \leq m_{H^{ \pm}} \leq 90 \mathrm{GeV}$. However, we are not aware of any LHC simulations, and so at present, it is not clear whether or not this strategy could give a sensitivity that is competitive with that for the appearance searches.

\section{LEP2 searches and future $e^{+} e^{-}$colliders}

The production mechanism at LEP2 was $e^{+} e^{-} \rightarrow$ $H^{+} H^{-}$. An important difference with the searches for $H^{ \pm}$at hadron colliders is that the couplings $X, Y, Z$ do not appear in the production cross section for $e^{+} e^{-} \rightarrow H^{+} H^{-}$, which is instead a function of just one unknown parameter $m_{H^{ \pm}}$. Hence, this production mechanism at $e^{+} e^{-}$colliders can produce $H^{ \pm}$even with very small values of $X, Y, Z$, provided that $2 m_{H^{ \pm}}<\sqrt{s}$.

The LEP working group combined the separate searches from the four LEP experiments [46]. These searches were carried out at energies in the range $\sqrt{s}=183 \mathrm{GeV}$ to $\sqrt{s}=209 \mathrm{GeV}$, and with a total combined integrated luminosity of $2.6 \mathrm{fb}^{-1}$. In the searches for the fermionic decay modes of $H^{ \pm}$, it is assumed that $\operatorname{BR}\left(H^{ \pm} \rightarrow c s\right)+$ $\operatorname{BR}\left(H^{ \pm} \rightarrow \tau \nu\right)=1$, but the actual experimental search for $H^{ \pm} \rightarrow c s$ would be also be sensitive to $H^{ \pm} \rightarrow c b$ and other light flavors of quark. Dedicated searches for the decay mode $H^{ \pm} \rightarrow A^{0} W^{*}$ were also carried out in [46], but in this work we are assuming that this channel is absent or very suppressed. From the search for fermionic decays the excluded region at $95 \%$ confidence level (CL) in the plane $\left[m_{H^{ \pm}}, \operatorname{BR}\left(H^{ \pm} \rightarrow \tau \nu\right)\right]$ is shown. For $m_{H^{ \pm}}<80 \mathrm{GeV}$ the whole range $0 \leq \mathrm{BR}\left(H^{ \pm} \rightarrow \tau \nu\right) \leq 100 \%$ is excluded. For $80 \mathrm{GeV} \leq m_{H^{ \pm}}<90 \mathrm{GeV}$, most of the region is not excluded for $\mathrm{BR}\left(H^{ \pm} \rightarrow \tau \nu\right)<80 \%$ (i.e., $\mathrm{BR}\left(H^{ \pm} \rightarrow c s\right)>$ $20 \%)$. Notably, there is an excess of events of greater than $2 \sigma$ significance around the point $m_{H^{ \pm}}=89 \mathrm{GeV}$, $\operatorname{BR}\left(H^{ \pm} \rightarrow c s\right)=65 \%$ and $\operatorname{BR}\left(H^{ \pm} \rightarrow \tau \nu\right)=35 \%$, which could be readily accommodated in a $3 \mathrm{HDM}$ with appropriate choices of $X, Y$ and $Z$. As mentioned in our earlier work [10] an excess like this is an example of a possible signal for $H^{ \pm}$that was just out of the range of LEP2. Such an excess, if genuine, could be observed at the LHC provided that the values of $|X|$ and $|Y|$ are large enough to ensure enough events of $t \rightarrow H^{ \pm} b$ at a given integrated luminosity. Future LHC searches in the $\tau \nu$ channel, which currently have sensitivity to the region $80 \mathrm{GeV} \leq m_{H^{ \pm}} \leq$ $90 \mathrm{GeV}$, could then observe such an $H^{ \pm}$. One could also expect a signal in the $H^{ \pm} \rightarrow c s / c b$ channel provided that sensitivity to the region $80 \mathrm{GeV} \leq m_{H^{ \pm}} \leq 90 \mathrm{GeV}$ is obtained. If $|X|$ and $|Y|$ are sufficiently small then such an $H^{ \pm}$would escape detection at the LHC, but could be observed at future $e^{+} e^{-}$colliders (see below).

The possibility of a future circular $e^{+} e^{-}$collider operating at a variety of energies from $\sqrt{s}=m_{Z}$ to $\sqrt{s}=2 m_{t}$ is being discussed (FCC-ee at CERN and CEPC in China), and a future $e^{+} e^{-}$Linear Collider (ILC) would also take data in this energy range (and higher energies). If such machines are approved, the earliest starting date of operation for CEPC (FCC-ee) would be the year 2030 (2040), with the ILC possibly starting between these two dates. The choice of $\sqrt{s}=240 \mathrm{GeV}$ would be optimal for detailed studies of the discovered $125 \mathrm{GeV}$ neutral boson. This energy would also enable pair production of $H^{ \pm}$up to a mass of $120 \mathrm{GeV}$. The integrated luminosity with $\sqrt{s}=$ $240 \mathrm{GeV}$ at all three colliders is expected to be of the order of a few $\mathrm{ab}^{-1}$, which is three orders of magnitude greater than the integrated luminosity $\left(2.6 \mathrm{fb}^{-1}\right)$ used in the combined LEP search in [46]. Hence, an $H^{ \pm}$with a mass in the region $80 \mathrm{GeV} \leq m_{H^{ \pm}}<90 \mathrm{GeV}$ would be discovered for any value of $\operatorname{BR}\left(H^{ \pm} \rightarrow c s / c b\right)$, with a signal in at least one of the three channels $H^{+} H^{-} \rightarrow j j j j, j j \tau \nu, \tau \nu \tau \nu$ (where $j$ signifies quarks lighter than the $t$ quark). As mentioned earlier, the production mechanism $e^{+} e^{-} \rightarrow$ $\mathrm{H}^{+} \mathrm{H}^{-}$does not depend on the couplings to fermions. Hence, an $H^{ \pm}$with $2 m_{H^{ \pm}}<\sqrt{s}$ that escaped detection at the LHC due to small values of $X, Y$, and $Z$ would be discovered at the above $e^{+} e^{-}$colliders.

\section{RESULTS}

We vary the four input parameters that determine $X, Y$, and $Z$ in the following ranges (see e.g., [14]):

$$
\begin{array}{cc}
-\pi / 2 \leq \theta \leq 0, & 0 \leq \delta \leq 2 \pi, \\
1 \leq \tan \beta \leq 60, & 1 \leq \tan \gamma \leq 60 .
\end{array}
$$

We have checked that the phenomenological constraints on $|X|,|Y|,|Z|$, and $|X Z|$ from Sec. II B are respected but we do not show explicit plots for these parameters. The constraints on $\operatorname{Re}\left(X Y^{*}\right)$ and $\operatorname{Im}\left(X Y^{*}\right)$ rule out significant regions of parameter space, and these will be shown in specific plots. Taking $\delta=0$ leads to real values for $X, Y$, and $Z$, and so in this case the constraint on $\operatorname{Im}\left(X Y^{*}\right)$ will be automatically respected. We only consider $m_{H^{ \pm}}<m_{t}$, and results will be presented for the cases of $m_{H^{ \pm}}=85 \mathrm{GeV}$ (for which the LHC currently has no sensitivity if $\operatorname{BR}\left(H^{ \pm} \rightarrow c s / c b\right)$ is dominant) and $m_{H^{ \pm}}=130 \mathrm{GeV}$ (for which the LHC has already set limits). We pick $m_{H^{ \pm}}=$ $85 \mathrm{GeV}$ as a representative choice that is midway in the 

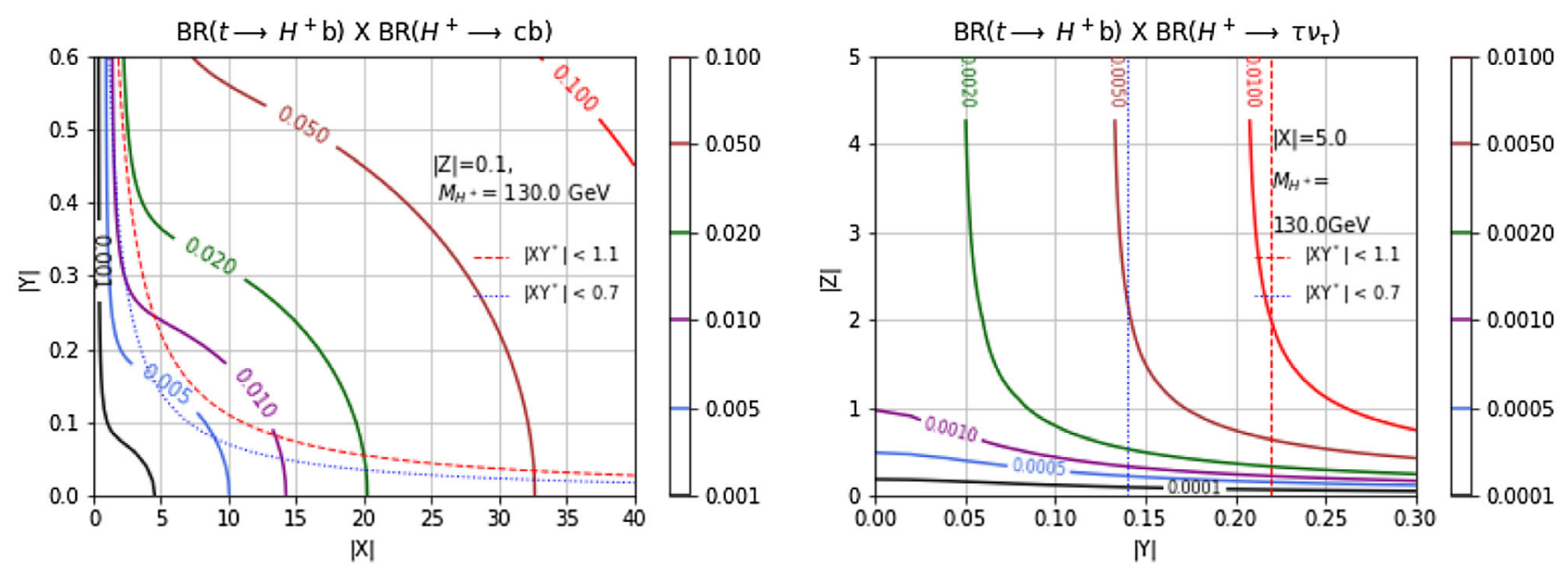

FIG. 1. Left panel: Contours of $\mathrm{BR}\left(t \rightarrow H^{ \pm} b\right) \times \mathrm{BR}\left(H^{ \pm} \rightarrow c b\right)$ in the plane $[|X|,|Y|]$ with $m_{H^{ \pm}}=130 \mathrm{GeV}$ and $|Z|=0.1$. Right panel: Contours of $\operatorname{BR}\left(t \rightarrow H^{ \pm} b\right) \times \operatorname{BR}\left(H^{ \pm} \rightarrow \tau \nu\right)$ in the plane $[|Y|,|Z|]$ with $m_{H^{ \pm}}=130 \mathrm{GeV}$ and $|X|=5$.

interval $80 \mathrm{GeV} \leq m_{H^{ \pm}} \leq 90 \mathrm{GeV}$, although our results will apply to all values of $m_{H^{ \pm}}$in this interval with small numerical differences. The searches at LEP [46] cannot rule out $\mathrm{BR}\left(H^{ \pm} \rightarrow c s\right) \geq 50 \%$ for $80 \mathrm{GeV} \leq m_{H^{ \pm}} \leq 83 \mathrm{GeV}$ and $88 \mathrm{GeV} \leq m_{H^{ \pm}} \leq 90 \mathrm{GeV}$. However, in the interval $83 \mathrm{GeV} \leq m_{H^{ \pm}} \leq 88 \mathrm{GeV}$ the values $\operatorname{BR}\left(H^{ \pm} \rightarrow c s\right) \geq$ $90 \%$ are ruled out (but $50 \% \leq \mathrm{BR}\left(H^{ \pm} \rightarrow c s\right) \leq 90 \%$ are not). We do not impose this small excluded region on our figures.

In our numerical analysis, we are only concerned with the four parameters in Eq. (12) and $m_{H^{ \pm}}$. These comprise five of the sixteen ${ }^{1}$ free parameters in the scalar potential of the 3HDM [14]. There are theoretical constraints on these sixteen parameters from requiring the stability of the vacuum, the absence of charge breaking minima, and compliance with unitarity of scattering processes, etc. Such constraints are well known in the 2 HDM (e.g., see [47] for a recent study) and have been discussed for the scalar potential of the $3 \mathrm{HDM}$ in $[48,49]$.

In our analysis, we do not impose these constraints because they would only rule out certain regions of the parameter space of sixteen variables. As mentioned earlier, the phenomenology in the charged Higgs sector depends on only five parameters (which we take as unconstrained parameters in the above ranges). We assume that the freedom in the remaining eleven parameters can be used to comply with the above theoretical constraints while allowing the five parameters in the charged Higgs sector to vary in the above ranges. To justify this approach we note that the analogous constraints on the scalar potential in 2HDMs do not restrict the allowed ranges of the two parameters in the charged Higgs sector $\left(m_{H^{ \pm}}\right.$and $\left.\tan \beta\right)$ due to the freedom in the remaining four parameters (for the

\footnotetext{
${ }^{1}$ There are originally eighteen free parameters in the scalar potential of the $3 \mathrm{HDM}$, but two are determined by the mass of the $W$ boson and the mass of the $125 \mathrm{GeV}$ neutral Higgs boson.
}

case of a $2 \mathrm{HDM}$ scalar potential with only soft breaking terms of a $Z_{2}$ symmetry). It is experimental data from processes involving $H^{ \pm}$that constrain the ranges of the parameters of the charged Higgs sector in a 2HDM, and we carry this conclusion across to the charged Higgs sector of the 3HDM.

In the left panel of Fig. 1, we show contours of $\mathrm{BR}\left(t \rightarrow H^{ \pm} b\right) \times \mathrm{BR}\left(H^{ \pm} \rightarrow c b\right)$ in the plane $[|X|,|Y|]$ for $|Z|=0.1$ and $m_{H^{ \pm}}=130 \mathrm{GeV}$. This is an update of a figure in [9] in which the contours have been chosen to reflect the current and future sensitivity of the LHC. The region consistent with $b \rightarrow s \gamma$ lies below the curves of $\left|X Y^{*}\right| \leq 0.7$ or $\left|X Y^{*}\right| \leq 1.1$, depending on the sign of $\operatorname{Re}\left(X Y^{*}\right)$ in Eq. (6). In this figure, we take $|X|$ and $|Y|$ as independent parameters and thus we do not consider them to be functions of the four parameters in Eq. (12) as in a 3HDM. As mentioned at the end of Sec. II C, in models such as the aligned 2HDM and a 4HDM the parameters $X$, $Y$, and $Z$ would depend on more than four parameters. The results in the left panel of Fig. 1 are a model independent approach in which the allowed region of $[|X|,|Y|]$ (for a given $|Z|)$ are shown. For the chosen value of $m_{H^{ \pm}}=$ $130 \mathrm{GeV}$ the current limit on $\operatorname{BR}\left(t \rightarrow H^{ \pm} b\right) \times \operatorname{BR}\left(H^{ \pm} \rightarrow\right.$ $c b)$ is $\leq 0.005$ [12]. It can be seen from the left panel of Fig. 1 that the current limit is ruling out parameter space that is permitted by $b \rightarrow s \gamma$. The contour with 0.001 will hopefully be approached with $150 \mathrm{fb}^{-1}$ at $\sqrt{s}=13 \mathrm{TeV}$, and such a search would further probe parameter space of $[|X|,|Y|]$, for a given $|Z|$, that is still allowed by $b \rightarrow s \gamma$.

In the right panel of Fig. 1, we show contours of $\operatorname{BR}(t \rightarrow$ $\left.H^{ \pm} b\right) \times \mathrm{BR}\left(H^{ \pm} \rightarrow \tau \nu\right)$ in the plane $[|Y|,|Z|]$ for $|X|=5$. This is also a model independent approach, and such a plot was not shown in [9]. In this case, the region allowed by $b \rightarrow s \gamma$ lies to the left of the perpendicular lines. For the chosen value of $m_{H^{ \pm}}=130 \mathrm{GeV}$ the current limit on $\mathrm{BR}\left(t \rightarrow H^{ \pm} b\right) \times \mathrm{BR}\left(H^{ \pm} \rightarrow \tau \nu\right)$ is $\leq 0.001$ [44]. It can be seen from the right panel of Fig. 1 that the current 

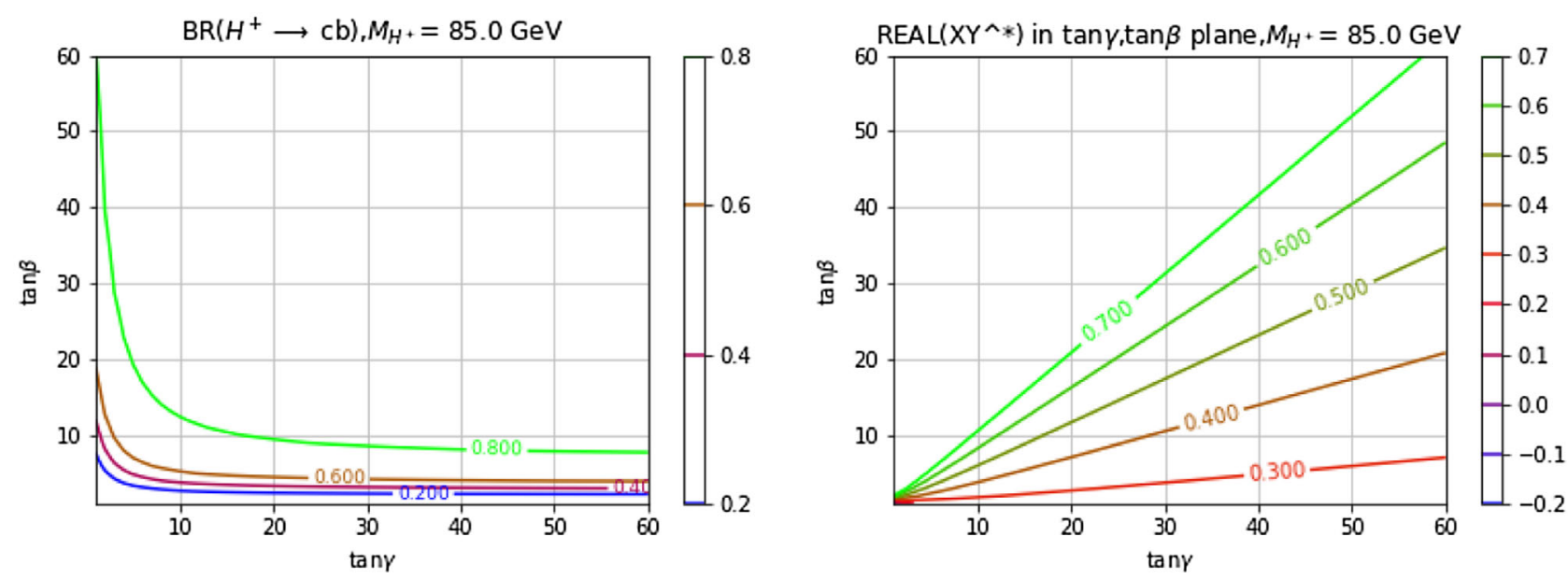

FIG. 2. The flipped $3 \mathrm{HDM}$ with $\theta=-\pi / 3, \delta=0$, and $m_{H^{ \pm}}=85 \mathrm{GeV}$. Left panel: Contours of $\mathrm{BR}\left(H^{ \pm} \rightarrow c b\right)$ in the plane $[\tan \gamma, \tan \beta]$. Right panel: Contours of $\operatorname{Re}\left(X Y^{*}\right)$ in the plane $[\tan \gamma, \tan \beta]$. The allowed parameter space lies below the contour of $\operatorname{Re}\left(X Y^{*}\right)=0.7$.
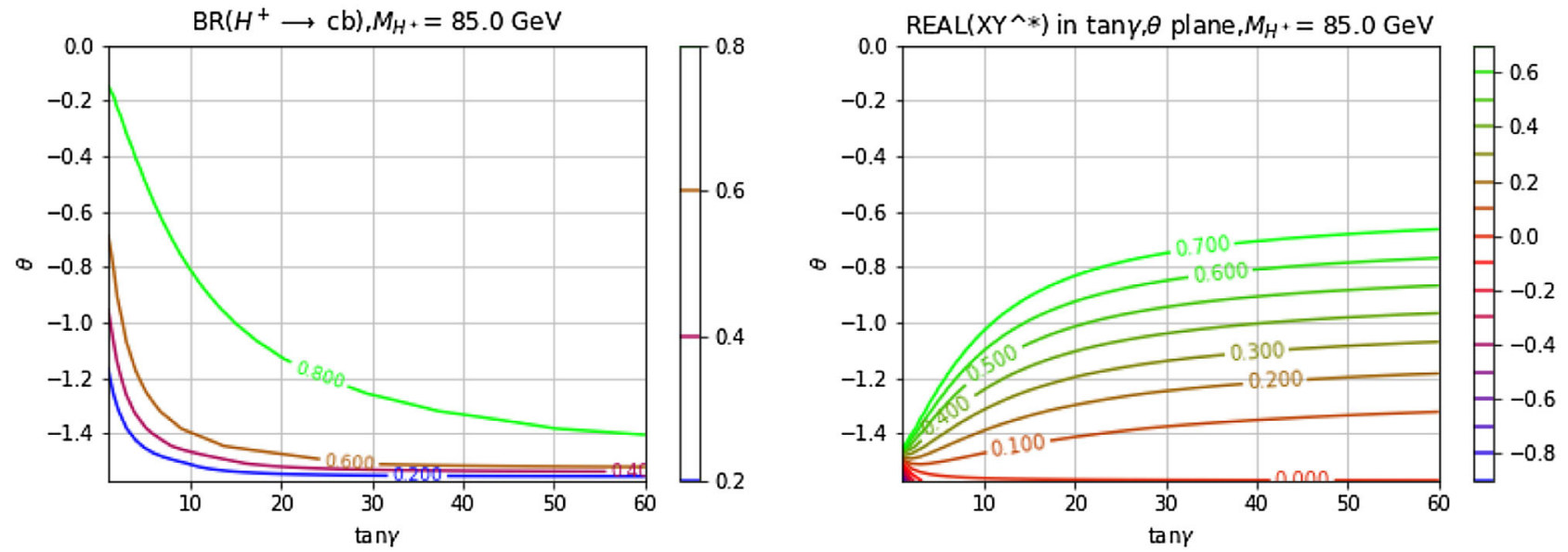

FIG. 3. The flipped 3HDM with $\tan \beta=10, \delta=0$, and $m_{H^{ \pm}}=85 \mathrm{GeV}$. Left panel: Contours of $\mathrm{BR}\left(H^{ \pm} \rightarrow c b\right)$ in the plane $[\tan \gamma, \theta]$. Right panel: Contours of $\operatorname{Re}\left(X Y^{*}\right)$ in the plane $[\tan \gamma, \theta]$. The allowed parameter space lies below the contour of $\operatorname{Re}\left(X Y^{*}\right)=0.7$.

limit is ruling out large regions of parameter space that is permitted by $b \rightarrow s \gamma$. The contours with 0.005 and below will hopefully be approached with $150 \mathrm{fb}^{-1}$ at $\sqrt{s}=13 \mathrm{TeV}$, and such a search would further probe parameter space of $[|Y|,|Z|]$, for a given $|X|$, that is still allowed by $b \rightarrow s \gamma$.

We now show our results for the flipped 3HDM and the democratic 3HDM. In the other 3HDMs (type I, type II, and Lepton-specific), we have checked that a large $\operatorname{BR}\left(H^{ \pm} \rightarrow\right.$ $c b)$ is not possible, and the maximum value is typically of the order of a few percent. In Fig. 2, we consider the flipped $3 \mathrm{HDM}$ with $\theta=-\pi / 3, \delta=0$, and $m_{H^{ \pm}}=85 \mathrm{GeV}$. In the left panel of Fig. 2, we show contours of $\operatorname{BR}\left(H^{ \pm} \rightarrow c b\right)$ in the plane $[\tan \gamma, \tan \beta]$. It is evident that for $\tan \gamma \geq 5$ and $\tan \beta \geq 5$ one has $\operatorname{BR}\left(H^{ \pm} \rightarrow c b\right) \geq 60 \%$, and for $\tan \gamma \geq$ 10 and $\tan \beta \geq 10$ the maximum value of around $80 \%$ is obtained. However, not all of this parameter space of $[\tan \gamma, \tan \beta]$ survives the constraint from $b \rightarrow s \gamma$. This can be seen in the right panel of Fig. 2 in which we show contours of $\operatorname{Re}\left(X Y^{*}\right)$ in the plane $[\tan \gamma, \tan \beta]$. The allowed parameter space lies below the contour of $\operatorname{Re}\left(X Y^{*}\right)=0.7$, and roughly corresponds to the parameter space of $\tan \gamma>\tan \beta$. By comparing the left and right panels it is clear that a large parameter space for a dominant $\mathrm{BR}\left(H^{ \pm} \rightarrow\right.$ $c b) \geq 60 \%$ (left panel) survives the $b \rightarrow s \gamma$ constraint (right panel). Taking a nonzero value of $\delta$ would only lead to slight modifications of $\operatorname{BR}\left(H^{ \pm} \rightarrow c b\right)$, but would change the regions allowed by $b \rightarrow s \gamma$ due to $X$ and $Y$ both gaining an imaginary part. For $\delta=0$ the constraint in Eq. (7) from the electric dipole moment of the neutron is automatically satisfied. For $\delta \neq 0$ this latter constraint would rule out parameter space, and we will consider this scenario later for the democratic 3HDM. 

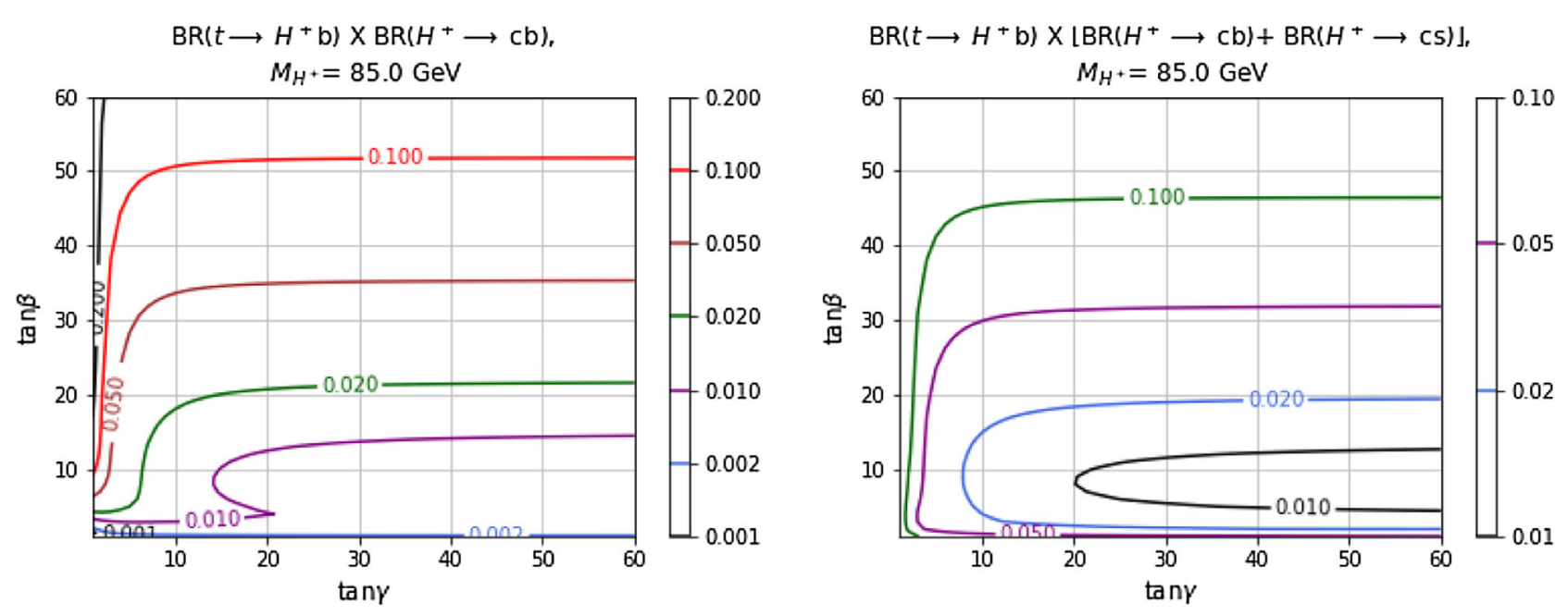

FIG. 4. The flipped 3HDM with $\theta=-\pi / 3, \delta=0$, and $m_{H^{ \pm}}=85 \mathrm{GeV}$. Left panel: Contours of $\mathrm{BR}\left(t \rightarrow H^{ \pm} b\right) \times \mathrm{BR}\left(H^{ \pm} \rightarrow c b\right)$ in the plane $[\tan \gamma, \tan \beta]$. Right panel: Contours of $\mathrm{BR}\left(t \rightarrow H^{ \pm} b\right) \times\left[\mathrm{BR}\left(H^{ \pm} \rightarrow c b\right)+\mathrm{BR}\left(H^{ \pm} \rightarrow c s\right)\right]$ in the plane $[\tan \gamma, \tan \beta]$.

In Fig. 3, we consider the flipped 3HDM with $m_{H^{ \pm}}=$ $85 \mathrm{GeV}$ but now with $\tan \beta=10$ and $\delta=0$. In the left panel of Fig. 3, we show contours of $\mathrm{BR}\left(H^{ \pm} \rightarrow c b\right)$ in the plane $[\tan \gamma, \theta]$. In the right panel of Fig. 3, we show contours of $\operatorname{Re}\left(X Y^{*}\right)$ in the plane $[\tan \gamma, \theta]$. There is a large parameter space for a dominant $\operatorname{BR}\left(H^{ \pm} \rightarrow c b\right)$ which corresponds to large values of $\tan \gamma$ and less negative values of $\theta$. In the right panel of Fig. 3, the parameter space allowed by $b \rightarrow s \gamma$ lies below the contour of $\operatorname{Re}\left(X Y^{*}\right)=0.7$, and thus a large parameter space for a dominant $\operatorname{BR}\left(H^{ \pm} \rightarrow c b\right) \geq 60 \%$ (left panel) survives the $b \rightarrow s \gamma$ constraint (right panel). In summary, from the results in Figs. 2 and 3, it is clear that a large part of the $[\tan \gamma, \tan \beta, \theta]$ parameter space (with $\delta=0$ ) gives rise to a dominant $\operatorname{BR}\left(H^{ \pm} \rightarrow c b\right)$ while complying with constraints from $b \rightarrow s \gamma$. As mentioned earlier, we consider the right panels of Figs. 2 and 3 to be representative of the true constraints on the planes $[\tan \gamma, \tan \beta]$ and $[\tan \gamma, \theta]$ from $b \rightarrow s \gamma$. We expect that the true excluded region would be shifted somewhat from the excluded regions in Figs. 2 and 3 , but it would not increase significantly in area. Given the large parameter space for a dominant $\operatorname{BR}\left(H^{ \pm} \rightarrow c b\right)$ in the flipped 3HDM we expect a sizeable region of large BR to survive. Taking a nonzero value of $\delta$ would only lead to slight modifications of the above plots for $\operatorname{BR}\left(H^{ \pm} \rightarrow c b\right)$, but would have an effect on the plot for $\operatorname{Re}\left(X Y^{*}\right)$. We will illustrate this when we consider the democratic 3HDM below.

In Fig. 4, we take the input parameters of Fig. 2 for the flipped 3HDM. In the left panel, we plot contours of $\mathrm{BR}\left(t \rightarrow H^{ \pm} b\right) \times \mathrm{BR}\left(H^{ \pm} \rightarrow c b\right)$ in the plane $[\tan \beta, \tan \gamma]$. This is the product that is being constrained by the CMS search at the LHC using three $b$-tags [12]. However, for $m_{H^{ \pm}}=85 \mathrm{GeV}$ [which is used in the Fig. 4] there is no limit on $\operatorname{BR}\left(t \rightarrow H^{ \pm} b\right) \times \mathrm{BR}\left(H^{ \pm} \rightarrow c b\right)$ from the LHC. The only limit is $\leq 20 \%$ from the Tevatron [34], using a strategy that was sensitive to any quark decay mode of $H^{ \pm}$. We plot contours of $\mathrm{BR}\left(t \rightarrow H^{ \pm} b\right) \times \mathrm{BR}\left(H^{ \pm} \rightarrow c b\right)$ with values of 0.2 to 0.002 . The region of the $[\tan \beta, \tan \gamma]$ plane that is above the contour of 0.2 is ruled out, while the region below corresponds to a potential discovery of such an $H^{ \pm}$. It is hoped that future searches of the LHC with $\sqrt{s}=$ $13 \mathrm{TeV}$ and $150 \mathrm{fb}^{-1}$ (or more) of data will have sensitivity to $\mathrm{BR}\left(t \rightarrow H^{ \pm} b\right) \times \mathrm{BR}\left(H^{ \pm} \rightarrow c b\right)$ of 0.02 or below.

In the right panel of Fig. 4, contours of $\operatorname{BR}\left(t \rightarrow H^{ \pm} b\right) \times$ $\mathrm{BR}\left[\left(H^{ \pm} \rightarrow c b\right)+\mathrm{BR}\left(H^{ \pm} \rightarrow c s\right)\right]$ are plotted with $m_{H^{ \pm}}=$ $85 \mathrm{GeV}$. This product is the observable that is being constrained by the searches that use $2 b$ tags $[38,42]$, and the figure is very similar to the left panel of Fig. 4. However, to obtain sensitivity to a given contour we expect that the $2 b$ search will require more integrated luminosity than the $3 b$ search, because the latter has smaller backgrounds as discussed earlier in Sec. III B 2.

Figure 5 is the same as Fig. 4 but with $m_{H^{ \pm}}=130 \mathrm{GeV}$, and hence $\operatorname{BR}\left(t \rightarrow H^{ \pm} b\right)$ is reduced compared to the corresponding case with $m_{H^{ \pm}}=85 \mathrm{GeV}$. However, in both panels in Fig. 5, the current excluded region is roughly above the contour of 0.02 (instead of 0.2 ) due to the LHC searches $[12,38,42]$ having superior sensitivity to those of the Tevatron in the region $90 \mathrm{GeV} \leq m_{H^{ \pm}} \leq 160 \mathrm{GeV}$. It can be seen that a sizeable area of the $[\tan \gamma, \tan \beta]$ parameter space is ruled out, while the region below the 0.02 contour would provide a possible signal for $H^{ \pm}$. It is hoped that future searches will have sensitivity to contours of 0.001 in both the $2 b$ and $3 b$ channels for $90 \mathrm{GeV} \leq$ $m_{H^{ \pm}} \leq 160 \mathrm{GeV}$.

We now show results in the democratic 3HDM. Taking $\delta=0$ we find that large values of $\operatorname{BR}\left(H^{ \pm} \rightarrow c b\right)$ are possible in regions of parameter space, but these regions are essentially ruled out by the $b \rightarrow s \gamma$ constraint. However, by taking $\delta \neq 0$ there are regions that have a large $\operatorname{BR}\left(H^{ \pm} \rightarrow c b\right)$ while complying with the constraints from 

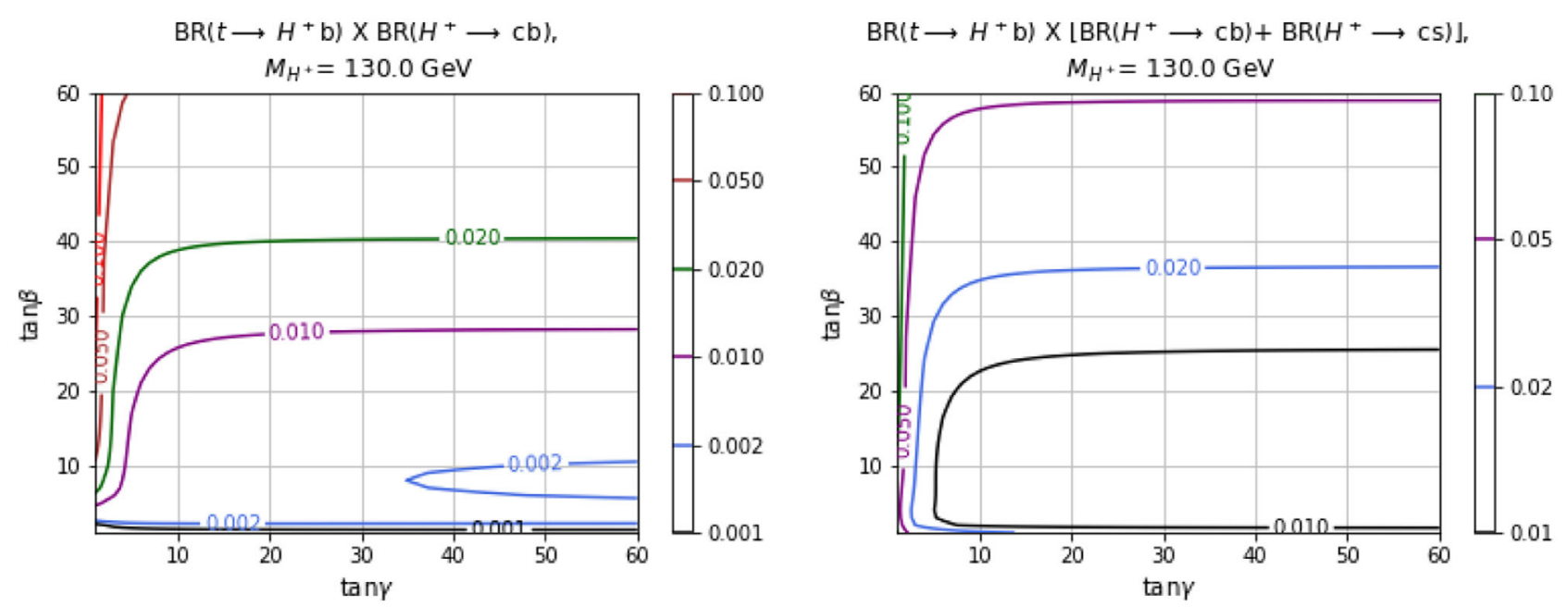

FIG. 5. The flipped 3HDM with $\theta=-\pi / 3, \delta=0$, and $m_{H^{ \pm}}=130 \mathrm{GeV}$. Left panel: Contours of $\mathrm{BR}\left(t \rightarrow H^{ \pm} b\right) \times \mathrm{BR}\left(H^{ \pm} \rightarrow c b\right)$ in the plane $[\tan \gamma, \tan \beta]$. Right panel: Contours of $\operatorname{BR}\left(t \rightarrow H^{ \pm} b\right) \times\left[\mathrm{BR}\left(H^{ \pm} \rightarrow c b\right)+\mathrm{BR}\left(H^{ \pm} \rightarrow c s\right)\right]$ in the plane $[\tan \gamma, \tan \beta]$.
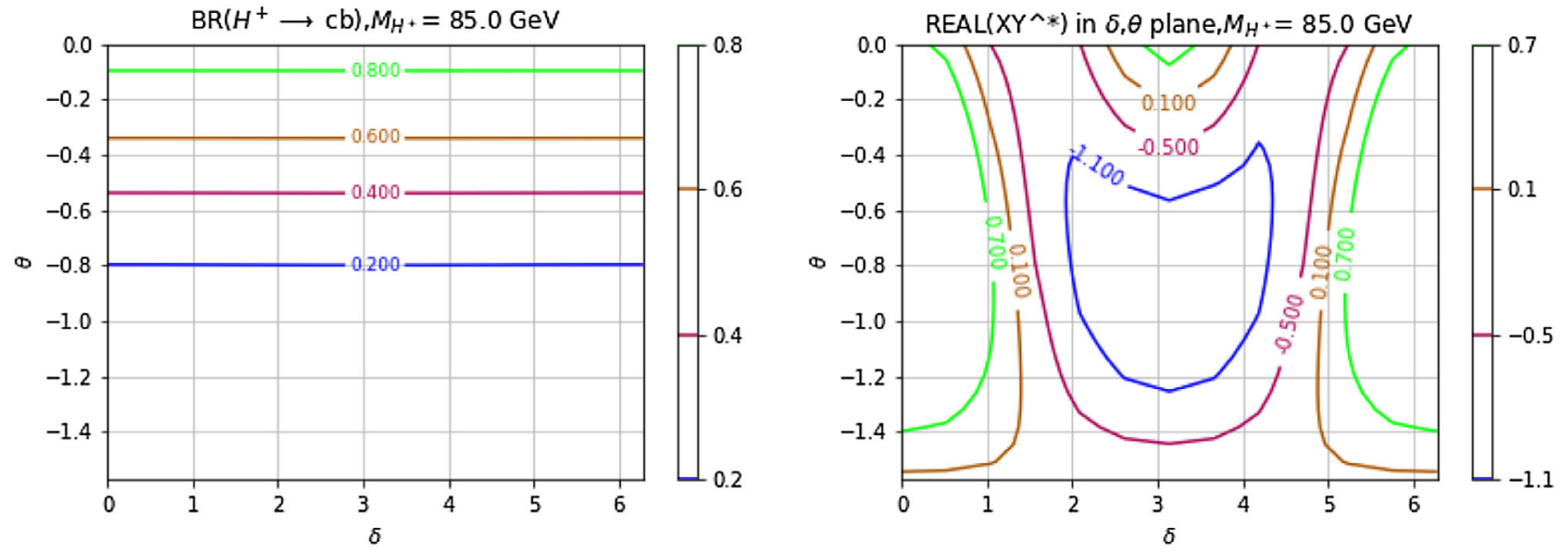

FIG. 6. The democratic 3HDM with $\tan \beta=40, \tan \gamma=10$, and $m_{H^{ \pm}}=85 \mathrm{GeV}$. Left panel: Contours of $\mathrm{BR}\left(H^{ \pm} \rightarrow c b\right)$ in the plane $[\delta, \theta]$. Right panel: Contours of $\operatorname{Re}\left(X Y^{*}\right)$ in the plane $[\delta, \theta]$. The allowed parameter space lies within the $\operatorname{range}-1.1 \leq \operatorname{Re}\left(X Y^{*}\right) \leq 0.7$.

$b \rightarrow s \gamma$ and the electric dipole moment of the neutron. In Fig. 6, we take $\tan \beta=40, \tan \gamma=10$, and $m_{H^{ \pm}}=85 \mathrm{GeV}$ in the democratic 3HDM. In the left panel, contours of $\mathrm{BR}\left(H^{ \pm} \rightarrow c b\right)$ are plotted in the plane $[\delta, \theta]$. It can be seen that large values of $\operatorname{BR}\left(H^{ \pm} \rightarrow c b\right)$ are possible, but $\delta$ has almost no effect on its magnitude. In the right panel of Fig. 6 , we plot contours of $\operatorname{Re}\left(X Y^{*}\right)$ in the plane $[\delta, \theta]$, and the allowed parameter space lies within the range $-1.1 \leq \operatorname{Re}\left(X Y^{*}\right) \leq 0.7$. One can see that varying $\delta$ has a sizeable effect on $\operatorname{Re}\left(X Y^{*}\right)$. By comparing the left and right panels it can be seen that the region $1 \leq \delta \leq 5$ and $0 \geq$ $\theta \geq-0.5$ gives a large $\operatorname{BR}\left(H^{ \pm} \rightarrow c b\right)$ that is also compatible with the $b \rightarrow s \gamma$ constraint. However, this region is further constrained by Fig. 7 in which we plot contours of $\operatorname{Im}\left(X Y^{*}\right)$ in the plane $[\delta, \theta]$, and the allowed parameter space lies within the range $\left|\operatorname{Im}\left(X Y^{*}\right)\right| \leq 0.1$. There are three allowed strips (with one being around $\delta=\pi$ ) in the

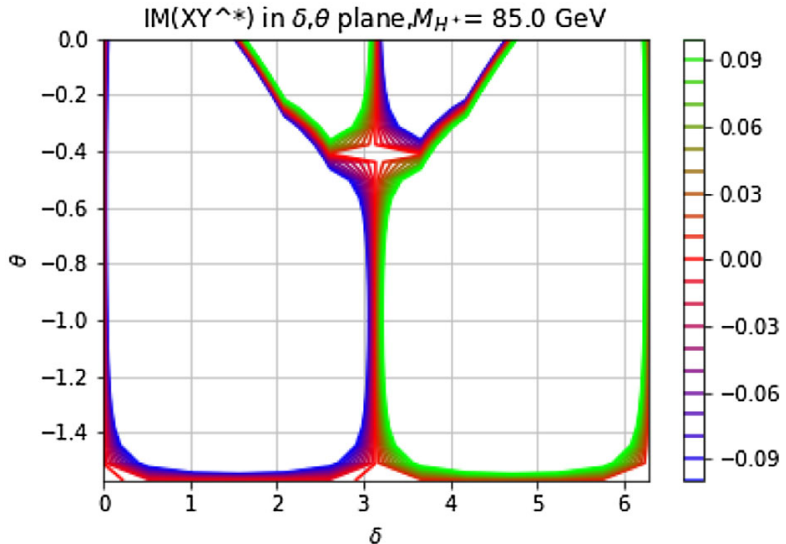

FIG. 7. The democratic 3HDM with $\tan \beta=40, \tan \gamma=10$, and $m_{H^{ \pm}}=85 \mathrm{GeV}$. Contours of $\operatorname{Im}\left(X Y^{*}\right)$ in the plane $[\delta, \theta]$. The allowed parameter space lies within the range $\left|\operatorname{Im}\left(X Y^{*}\right)\right|=0.1$. 

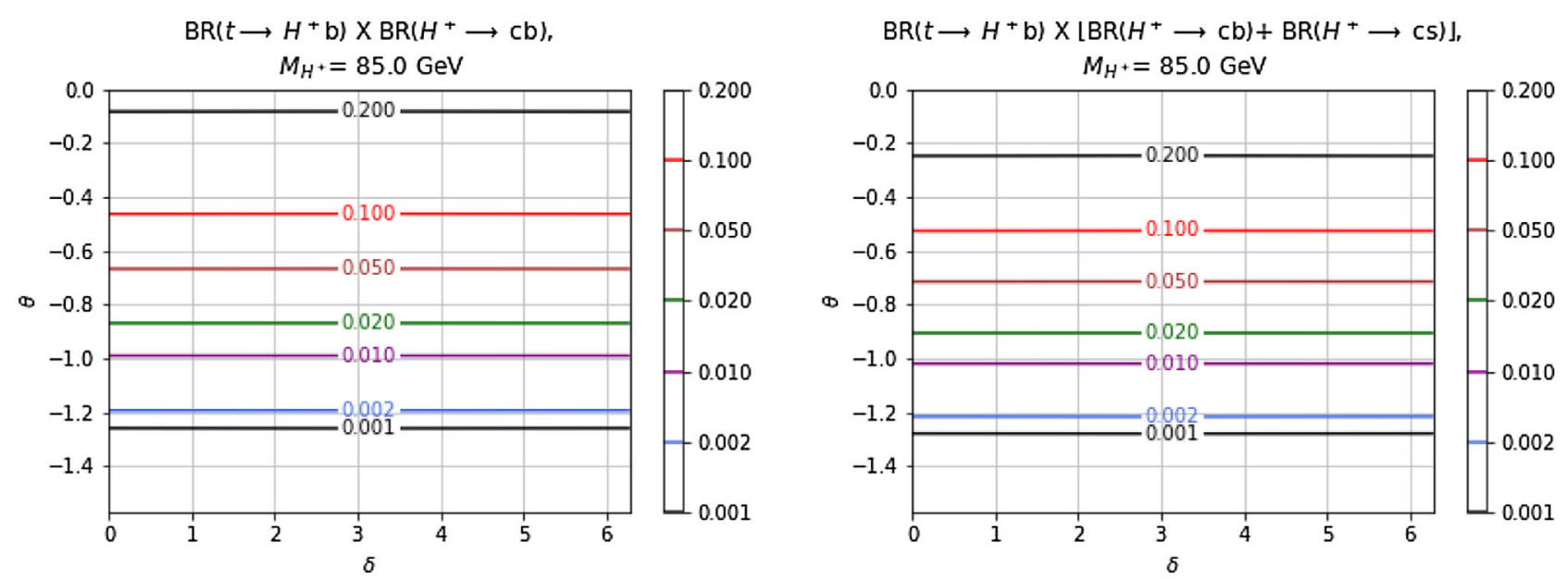

FIG. 8. The democratic $3 \mathrm{HDM}$ with $\tan \beta=40, \tan \gamma=10$, and $m_{H^{ \pm}}=85 \mathrm{GeV}$. Left panel: Contours of $\mathrm{BR}\left(t \rightarrow H^{ \pm} b\right) \times$ $\operatorname{BR}\left(H^{ \pm} \rightarrow c b\right)$ in the plane $[\theta, \delta]$. Right panel: Contours of $\operatorname{BR}\left(t \rightarrow H^{ \pm} b\right) \times\left[\operatorname{BR}\left(H^{ \pm} \rightarrow c b\right)+\operatorname{BR}\left(H^{ \pm} \rightarrow c s\right)\right]$ in the plane $[\theta, \delta]$.
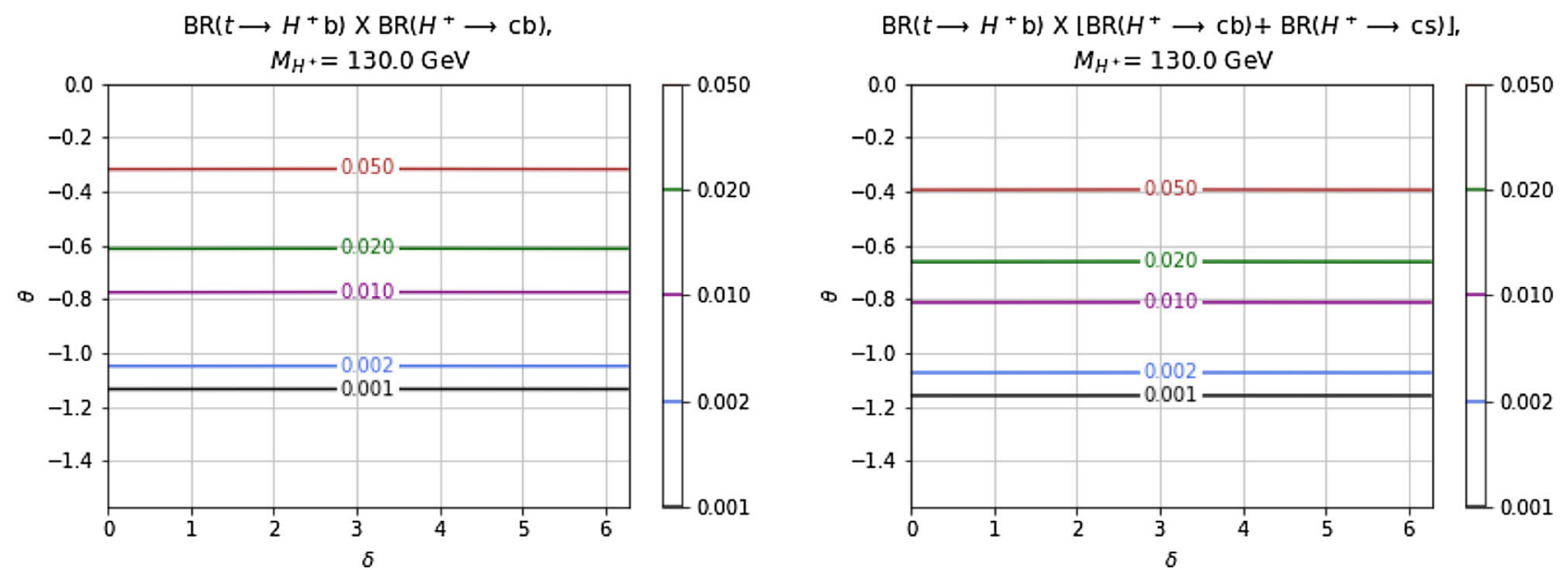

FIG. 9. The democratic $3 \mathrm{HDM}$ with $\tan \beta=40, \tan \gamma=10$, and $m_{H^{ \pm}}=130 \mathrm{GeV}$. Left panel: Contours of BR $\left(t \rightarrow H^{ \pm} b\right) \times$ $\mathrm{BR}\left(H^{ \pm} \rightarrow c b\right)$ in the plane $[\theta, \delta]$. Right panel: Contours of $\operatorname{BR}\left(t \rightarrow H^{ \pm} b\right) \times\left[\operatorname{BR}\left(H^{ \pm} \rightarrow c b\right)+\operatorname{BR}\left(H^{ \pm} \rightarrow c s\right)\right]$ in the plane $[\theta, \delta]$.

region of large $\operatorname{BR}\left(H^{ \pm} \rightarrow c b\right)$ (i.e., $0 \geq \theta \geq-0.5$ ). Consequently, the democratic $3 \mathrm{HDM}$ is a candidate model for a possible signal in future $3 b$ searches for $H^{ \pm}$as carried out in [12], although the parameter space for a large $\operatorname{BR}\left(H^{ \pm} \rightarrow c b\right)$ is much smaller than that in the flipped 3HDM, and is likely to require $\delta \neq 0$.

Figures 8 and 9 are with the same parameter choice of $\tan \beta=40, \tan \gamma=10$ in the democratic 3HDM, and are the plots that correspond to Figs. 4 and 5 in the flipped 3HDM. Contours of $\operatorname{BR}\left(t \rightarrow H^{ \pm} b\right) \times \mathrm{BR}\left(H^{ \pm} \rightarrow c b\right)$ and $\quad \operatorname{BR}\left(t \rightarrow H^{ \pm} b\right) \times\left[\mathrm{BR}\left(H^{ \pm} \rightarrow c b\right)+\mathrm{BR}\left(H^{ \pm} \rightarrow c s\right)\right]$ are plotted in the plane $[\delta, \theta]$. The (small) allowed region can be read off from Fig. 7, most of it being around $\delta=\pi$, but with $-0.6 \leq \theta \leq-1.2$ being excluded from Fig. 6 .

\section{CONCLUSIONS}

In summary, we have studied a $3 \mathrm{HDM}$ wherein two charged Higgs bosons states exist, one of which we have assumed to be lighter than the top quark and the other one heavier. Hence, the light state can be produced in (anti)top decays via $t \rightarrow b H^{ \pm}$, particularly at hadron colliders like the LHC, via the $p p \rightarrow t \bar{t}$ process, which herein has a significant cross section (nearing the nb level) so that the main focus of our analysis has been on this $H^{ \pm}$production channel. Amongst the possible $H^{ \pm}$decay modes in a 3HDM we have selected here the fermionic ones, i.e., $H^{ \pm} \rightarrow c s, c b$, and $\tau \nu$, which are those exploited in collider searches, at both past (LEP and Tevatron) and present (LHC) machines. Amongst these three channels, we have concentrated on $H^{ \pm} \rightarrow c b$ as it offers a twofold experimental advantage. On the one hand, the irreducible background from $W^{ \pm} \rightarrow c b$ decays is suppressed by the Cabibbo-Kobayashi-Maskawa (CKM) matrix. On the other hand, it can be filtered out by requiring a $b$-tag of one the two jets that eventually emerges in the detector. Furthermore, from a theoretical point of view this decay 
mode may be a privileged probe of the underlying 3HDM structure. This is because the $\operatorname{BR}\left(H^{ \pm} \rightarrow c b\right)$ can be large in the flipped and democratic versions of the $3 \mathrm{HDM}$, but not in the type I, type II, and lepton-specific structures, while being compatible with experimental constraints, chiefly, those from $b \rightarrow s \gamma$.

We have then performed the first comprehensive study of the decay mode $H^{ \pm} \rightarrow c b$ in terms of the four fundamental parameters of the charged Higgs sector of the $3 \operatorname{HDM}(\beta, \gamma$, $\theta$, and $\delta$ ) over the available $m_{H^{ \pm}}$range. We found that the parameter space for a large $\operatorname{BR}\left(H^{ \pm} \rightarrow c b\right)$ is much bigger in the flipped 3HDM than in the democratic 3HDM. Our emphasis has been on the interval $80 \mathrm{GeV}<m_{H^{ \pm}}<$ $90 \mathrm{GeV}$, to which the LHC has no sensitivity at present, the reason being that no experimental searches have yet been attempted for the decays $H^{ \pm} \rightarrow c b / c s$ at this collider. For the purpose of encouraging such searches, we have mapped out the 3HDM parameter spaces of the flipped and democratic types that can be accessible at the LHC as a function of its increased luminosity, concluding that they should be accessible in the near future by exploiting established experimental techniques. In fact, this can be achieved by resorting to both appearance and disappearance searches. The former would have direct sensitivity to the $H^{ \pm} \rightarrow c b$ channel while the latter would have indirect access one to it, via the absence of the expected number of $W^{ \pm} \rightarrow \ell \nu(\ell=e, \mu)$ and $W \rightarrow q \bar{q}$ events originating from $p p \rightarrow t \bar{t}$ with standard top decay for both $t$ and $\bar{t}$. Similarly positive prospects are expected for future $e^{+} e^{-}$colliders, like FCC-ee, CEPC, and ILC, where the $H^{ \pm}$state would be pair produced via $e^{+} e^{-} \rightarrow H^{+} H^{-}$. At such colliders the QCD backgrounds are much reduced with respect to those at the LHC, which greatly facilitates the extraction of the $H^{ \pm} \rightarrow c b$ mode.

\section{ACKNOWLEDGMENTS}

S. M. is funded in part through the NExT Institute, the STFC CG ST/L000296/1 and the H2020-MSCA-RISE2014 Grant No. 645722 (NonMinimalHiggs).
[1] G. Aad et al. (ATLAS Collaboration), Phys. Lett. B 716, 1 (2012).

[2] S. Chatrchyan et al. (CMS Collaboration), Phys. Lett. B 716, 30 (2012).

[3] M. Aaboud et al. (ATLAS Collaboration), Phys. Lett. B 786, 59 (2018).

[4] G. C. Branco, P. M. Ferreira, L. Lavoura, M. N. Rebelo, M. Sher, and J. P. Silva, Phys. Rep. 516, 1 (2012).

[5] A. G. Akeroyd et al., Eur. Phys. J. C 77, 276 (2017).

[6] Y. Grossman, Nucl. Phys. B426, 355 (1994).

[7] A. G. Akeroyd and W. J. Stirling, Nucl. Phys. B447, 3 (1995).

[8] A. G. Akeroyd, arXiv:hep-ph/9509203.

[9] A. G. Akeroyd, S. Moretti, and J. Hernandez-Sanchez, Phys. Rev. D 85, 115002 (2012).

[10] A. G. Akeroyd, S. Moretti, K. Yagyu, and E. Yildirim, Int. J. Mod. Phys. A 32, 1750145 (2017).

[11] S. L. Glashow and S. Weinberg, Phys. Rev. D 15, 1958 (1977); E. A. Paschos, Phys. Rev. D 15, 1966 (1977).

[12] A. M. Sirunyan et al. (CMS Collaboration), J. High Energy Phys. 11 (2018) 115.

[13] V. D. Barger, J. L. Hewett, and R. J. N. Phillips, Phys. Rev. D 41, 3421 (1990).

[14] G. Cree and H. E. Logan, Phys. Rev. D 84, 055021 (2011).

[15] M. Jung, A. Pich, and P. Tuzon, J. High Energy Phys. 11 (2010) 003.

[16] M. Trott and M. B. Wise, J. High Energy Phys. 11 (2010) 157.

[17] M. Ciuchini, E. Franco, G. Martinelli, L. Reina, and L. Silvestrini, Phys. Lett. B 334, 137 (1994).

[18] M. Ciuchini, G. Degrassi, P. Gambino, and G. F. Giudice, Nucl. Phys. B527, 21 (1998).
[19] F. Borzumati and C. Greub, Phys. Rev. D 58, 074004 (1998).

[20] P. Gambino and M. Misiak, Nucl. Phys. B611, 338 (2001).

[21] T. Hermann, M. Misiak, and M. Steinhauser, J. High Energy Phys. 11 (2012) 036.

[22] M. Misiak, H. M. Asatrian, R. Boughezal, M. Czakon, T. Ewerth, A. Ferroglia, P. Fiedler, P. Gambino et al., Phys. Rev. Lett. 114, 221801 (2015).

[23] S. Moretti and W. J. Stirling, Phys. Lett. B 347, 291 (1995); 366, 451(E) (1996).

[24] A. Djouadi, J. Kalinowski, and P. M. Zerwas, Z. Phys. C 70, 435 (1996).

[25] A. G. Akeroyd, Nucl. Phys. B544, 557 (1999).

[26] F. Kling, A. Pyarelal, and S. Su, J. High Energy Phys. 11 (2015) 051.

[27] A. Arhrib, R. Benbrik, and S. Moretti, Eur. Phys. J. C 77, 621 (2017).

[28] A. Arbey, F. Mahmoudi, O. Stal, and T. Stefaniak, Eur. Phys. J. C 78, 182 (2018).

[29] A. Arhrib, R. Benbrik, R. Enberg, W. Klemm, S. Moretti, and S. Munir, Phys. Lett. B 774, 591 (2017).

[30] M. Aoki, S. Kanemura, K. Tsumura, and K. Yagyu, Phys. Rev. D 80, 015017 (2009).

[31] H. E. Logan and D. MacLennan, Phys. Rev. D 81, 075016 (2010).

[32] A. Pich and P. Tuzon, Phys. Rev. D 80, 091702 (2009).

[33] J. Hernandez-Sanchez, S. Moretti, R. Noriega-Papaqui, and A. Rosado, J. High Energy Phys. 07 (2013) 044.

[34] V. M. Abazov et al. (D0 Collaboration), Phys. Lett. B 682, 278 (2009).

[35] T. Aaltonen et al. (CDF Collaboration), Phys. Rev. Lett. 103, 101803 (2009). 
[36] S. Chatrchyan et al. (CMS Collaboration), J. High Energy Phys. 07 (2012) 143.

[37] CMS Collaboration, CERN Report No. CMS-PAS-HIG-16031.

[38] G. Aad et al. (ATLAS Collaboration), Eur. Phys. J. C 73, 2465 (2013).

[39] G. Aad et al. (ATLAS Collaboration), J. High Energy Phys. 03 (2013) 076.

[40] G. Aad et al. (ATLAS Collaboration), J. High Energy Phys. 06 (2012) 039.

[41] G. Aad et al. (ATLAS Collaboration), J. High Energy Phys. 03 (2015) 088.

[42] V. Khachatryan et al. (CMS Collaboration), J. High Energy Phys. 12 (2015) 178.

[43] V. Khachatryan et al. (CMS Collaboration), J. High Energy Phys. 11 (2015) 018.
[44] M. Aaboud et al. (ATLAS Collaboration), J. High Energy Phys. 09 (2018) 139.

[45] CMS Collaboration, CERN Report No. CMS-PAS-HIG-18014.

[46] G. Abbiendi et al. (ALEPH, DELPHI, L3, OPAL, and LEP Collaborations), Eur. Phys. J. C 73, 2463 (2013).

[47] O. Eberhardt, U. Nierste, and M. Wiebusch, J. High Energy Phys. 07 (2013) 118.

[48] I. P. Ivanov, J. High Energy Phys. 07 (2010) 020; I. P. Ivanov and E. Vdovin, Phys. Rev. D 86, 095030 (2012); V. Keus, S. F. King, and S. Moretti, J. High Energy Phys. 01 (2014) 052; M. Maniatis and O. Nachtmann, J. High Energy Phys. 02 (2015) 058; 10 (2015) 149(E); S. Moretti and K. Yagyu, Phys. Rev. D 91, 055022 (2015).

[49] M. P. Bento, H. E. Haber, J. C. Romo, and J. P. Silva, J. High Energy Phys. 11 (2017) 095; H. E. Haber, O. M. Ogreid, P. Osland, and M. N. Rebelo, arXiv:1808.08629. 